\title{
Exploring the Performance-Controlling Tablet Disintegration Mechanisms for Direct Compression Formulations
}

\author{
Natalie Maclean ${ }^{\mathrm{a}}$, Erin Walsh ${ }^{\mathrm{a}, \mathrm{b}}$, Mithushan Soundaranathan $^{\mathrm{a}, \mathrm{b}}$, Ibrahim Khadra $^{\mathrm{a}}$, James Mann ${ }^{\mathrm{c}}$, \\ Helen Williams ${ }^{\mathrm{d}}$, Daniel Markl ${ }^{\mathrm{a}, \mathrm{b}, *}$ \\ ${ }^{a}$ Strathclyde Institute of Pharmacy $\&$ Biomedical Sciences, University of Strathclyde, Glasgow, UK \\ ${ }^{b}$ Future Continuous Manufacturing and Advanced Crystallisation Research Hub, University of Strathclyde, Glasgow, UK \\ ${ }^{c}$ Oral Product Development, Pharmaceutical Technology $\& 6$ Development, Operations, AstraZeneca, Macclesfield, UK \\ ${ }^{d}$ New Modalities and Parenteral Development, Pharmaceutical Technology 83 Development, Operations, AstraZeneca, \\ Macclesfield, UK
}

\begin{abstract}
The design and manufacture of tablets is a challenging process due to the complex interrelationships between raw material properties, the manufacturing setting and the tablet properties. An important factor in formulation and process design is the fact that raw material and tablet properties drive the disintegration and dissolution performance of the final drug product. This study aimed to identify the mechanisms which control tablet disintegration for 16 different immediate-release placebo formulations based on raw material and tablet properties. Each formulation consisted of two fillers (47\% each), one disintegrant and a lubricant. Tablets were manufactured by direct compression using four different combinations of the fillers microcrystalline cellulose (MCC), mannitol, lactose and dibasic calcium phosphate anhydrous (DCPA). The disintegration mechanism was primarily driven by the filler combination, where $\mathrm{MCC} /$ lactose tablets were identified as wettability controlled, MCC/mannitol tablets as dissolution controlled and DCPA-based tablets (MCC/DCPA and lactose/DCPA) as swelling controlled. A change of $2 \%$ in porosity for the wettability controlled tablets (MCC/lactose) caused a significant acceleration of the disintegration process $(77 \%$ reduction of disintegration time), whereas for swelling controlled tablets (MCC/DCPA) the same porosity change did not considerably impact the disintegration process (3\% change in disintegration time). By classifying these formulations, critical formulation and manufacturing properties can be identified to allow tablet performance to be optimised.
\end{abstract}

Keywords: disintegration mechanism, wettability, excipient swelling, dissolution, solid dosage form, formulation development

\section{Introduction}

The disintegration and dissolution performance of immediate-release (IR) tablets plays a central role in the therapeutic efficacy of a product. Before the active pharmaceutical ingredient (API) can have a therapeutic effect, it must first dissolve and then be absorbed into the systemic circulation. For most IR formulations, rapid disintegration is essential for dissolution. As the tablet breaks into smaller particles, the surface area available for dissolution increases, resulting in faster drug release.

${ }^{*}$ Corresponding Author: daniel.markl@strath.ac.uk
For a tablet to disintegrate, the repulsive forces within the tablet must exceed the inter-particle bonding forces. The first stage in tablet disintegration is liquid penetration. When in contact with liquid, the disintegration medium will penetrate the tablet through pores in the microstructure. Although liquid penetration is not directly responsible for disintegration, it is a prerequisite for all other disintegration mechanisms (Nogami et al., 1967). The most common cause of disintegration is the expansion of particles within the tablet. As the particles swell, the void space in the pores is soon filled and a force is exerted on the surrounding matrix. When this force exceeds the cohesive forces be- 
tween particles in the matrix, disintegration will occur (Caramella et al., 1990). Depending on the material, particle expansion can be omni-directional or uni-directional. The omni-directional expansion of particles is a result of moisture uptake and is typically referred to as swelling. On the other hand, some materials will only expand uni-directionally as a result of its deformation during compaction. For these materials, contact with liquid causes the particles to regain their original shape prior to compaction, and therefore expansion occurs only axially against the direction of compaction (Desai et al., 2012; Quodbach et al., 2014b). In this paper, this mechanism will be termed shape recovery, however it is also known as strain recovery or deformation recovery. Aside from swelling and shape recovery, disintegration can also be a result of the dissolution of particles from the matrix. Each of these mechanisms are influenced either by the raw material properties, manufacturing conditions or both (Markl and Zeitler, 2017).

For tablets manufactured by direct compression, the pore structure of the tablet is formed during compaction and this can impact the disintegration performance. Higher compression forces result in tablets with lower porosity. The tablet porosity influences the rate of liquid penetration into the tablet, and therefore the rate of disintegration (Patel and Hopponen, 1966; Marais et al., 2003). In addition to compression force, the disintegration performance of tablets manufactured by wet or dry granulation may also be influenced by granule density or moisture content or the mode of disintegrant addition (i.e. intra- or extra-granular) (Gordon et al., 1993).

The effect of formulation on tablet disintegration has been widely studied. In addition to the swelling and shape recovery mechanisms of disintegrants, other excipients in the formulation will also influence the disintegration process. Microcrystalline cellulose (MCC) is a popular filler used in direct compression, which swells when it comes in contact with liquid (Markl et al., 2017). It has been shown that the use of soluble fillers hinders disintegration compared to insoluble fillers (Johnson et al., 1991; Rubinstein and Birch, 1977; Berardi et al., 2018). As the soluble filler will begin to dissolve as liquid penetrates the tablet, the efficiency of swelling and shape recovery materials is reduced. With soluble of hygroscopic materials, there may be competition for water with the disintegrants (Johnson et al., 1991; Berardi et al., 2018). For insoluble ma- trices, the expansion of the disintegrant results in the disintegrating force being fully exerted on the surrounding particles (Rubinstein and Birch, 1977). Some studies have also suggested that dissolution of the filler will increase the viscosity of the medium and result in slower liquid penetration, however, these effects are likely to be small relative to the effect on disintegration time. Ekmekciyan et al. (2018) demonstrated that solubility effects can be explained by tablet components competing for the available water. Soluble binders and fillers were found to require more water molecules to dissolve, compared to insoluble fillers which can be fully wetted by a small amount of medium. As a result, the use of insoluble fillers leaves more water available for the disintegrants. These results supported previous studies which have shown that increased hygroscopicity of the tablet components could also result in decreased disintegration efficiency (Gordon and Chowhan, 1987; López-Solís and VillafuerteRobles, 2001; Johnson et al., 1991).

Disintegration testing is routinely performed throughout drug product development. However, the standard disintegration test gives very little mechanistic information about the disintegration of the tablet. As a result, several groups have applied novel approaches such as magnetic resonance imaging (Quodbach et al., 2014a,b; Dvořák et al., 2020), broadband acoustic resonance dissolution spectroscopy (BARDS) (O'Mahoney et al., 2020), image analysis (Berardi et al., 2018) and stress relaxation measurements (Tomas et al., 2018) which can be used to study the underlying processes occurring during disintegration and dissolution. Similarly, terahertz pulsed imaging (TPI) has been used to study liquid penetration in and swelling of powder compacts (Yassin et al., 2015; Markl et al., 2017; Al-Sharabi et al., 2020). The test conditions have also been shown to influence the disintegration and dissolution rates. Bisharat et al. (2019) investigated the role of ethanol on disintegrant efficiency, whilst Zhao and Augsburger (2005) explored the role of $\mathrm{pH}$ of the disintegrating medium. The disintegration process is also affected by the choice of dissolution medium (e.g. biorelevant medium) (Anwar et al., 2005) and its temperature (Basaleh et al., 2020). Several recent reviews have evaluated the current literature on the disintegration process, the measurement techniques available, and the mechanisms of action of tablet disintegrants (Quodbach and Kleinebudde, 2016; Desai et al., 2016; Markl and Zeitler, 2017). 
The objective of this study was to propose a workflow which could allow identification of the performance-controlling disintegration mechanism. In this study, a performance-controlling mechanism is referred to as a rate process that directly impacts the overall disintegration time. In other words, a minor alteration of one or multiple material and/or product attributes strongly affects the rate of the identified mechanism which in turn causes a change in the disintegration performance. This workflow is demonstrated for 16 different placebo formulations, each composed of commonly used excipients. Both raw material (e.g. moisture sorption, intrinsic dissolution rate) and tablet properties (e.g. porosity) were considered within the workflow. This study applies simple and routine analytical tests to gain a greater understanding of the formulation properties which affect disintegration. By applying these principles to understand the mechanism of a given formulation, it is possible to optimise formulation design in terms of the disintegration performance.

\section{Materials and Methods}

\subsection{Materials}

Microcrystalline cellulose (MCC) (Avicel@PH102, FMC International), mannitol (Pearlitol@200 SD, Roquette), lactose (FastFloß316, Foremost Farms USA) and dibasic calcium phosphate anhydrous (DCPA) (Anhydrous Emcompress@, JRS Pharma) were used as fillers for the tablet formulations. The disintegrants used were croscarmellose sodium (CCS) (FMC International), crospovidone (XPVP) (Kollidon@CL, BASF), low-substituted hydroxypropyl cellulose (L-HPC) (LH-21, ShinEtsu Chemical Co.) and sodium starch glycolate (SSG) (Primojel@), DFE Pharma). Magnesium stearate (Mallinckrodt) was used as a lubricant. All of the excipients used in this study were chosen due to their widespread use in industry. The fillers used in this study represent a broad range of physicochemical properties. MCC is a hygroscopic, insoluble filler which swells when in contact with liquid. DCPA is an insoluble filler which is nonhygroscopic. Lactose and mannitol are both soluble fillers. The disintegrants CCS, L-HPC and SSG are swelling disintegrants, whilst XPVP acts by shape recovery.

\subsection{Characterisation of Raw Materials}

\subsubsection{Dynamic Vapor Sorption}

Moisture sorption isotherms were collected by dynamic vapor sorption (DVS) at $25^{\circ} \mathrm{C}$ for each excipient. Samples of approximately $10 \mathrm{mg}$ were analysed using the DVS Advantage (Surface Measurement Systems, London, UK). Prior to analysis, samples were conditioned at $0 \%$ relative humidity ( $\mathrm{RH})$. After conditioning, the sample mass was recorded as the reference mass. The humidity was then increased in increments of $10 \% \mathrm{RH}$ until $90 \%$ RH. At each stage, the change in mass was recorded once the balance reading had stabilised to less than $0.002 \%$ change in mass per minute.

\subsubsection{Particle Size and Shape}

The shape and size of particles of each excipient was measured using a QICPIC instrument (Sympatec GmbH, Clausthal-Zellerfeld, Germany).

For each sample, the primary container was thoroughly mixed by rolling and inverting by hand, as well as using a spatula to stir. Sample sizes of approximately $2 \mathrm{~g}$ were gently agitated to evenly disperse the particles and reduce loss of material in the vials. Analysis was performed using the M7 lens. Each sample was measured in triplicate.

\subsubsection{True Density}

The true density of each excipient was measured using a gas pyncometer with nitrogen (MicroUltrapyc 1200e, Quantachrome instrument, Graz, Austria). Measurements were taken in triplicate for each sample.

\subsubsection{Single Particle Dissolution}

The dissolution of individual particles of lactose and mannitol was studied using a custom-built flow cell, as described by Soundaranathan et al. (2020). Briefly, particles are placed in a small spherical sample holder in the centre of the flow cell. A peristaltic pump was used to pump deionised water at $20^{\circ} \mathrm{C}$ through the flow cell at a rate of $1.73 \mathrm{~mL} / \mathrm{min}$. This process is recorded using an optical microscope (Leica DM6000, Leica Microsystems CMS GmbH, Germany) at 10x magnification. For each material, the dissolution of 12 particles was monitored.

\subsubsection{Relative Swelling}

The relative swelling for each formulation was calculated using the average particle size $\left(D_{50}\right)$ and 
the maximum swelling capacity $\left(\Delta r_{\max }\right)$. The measurement of $\Delta r_{\max }$ is described by Soundaranathan et al. (2020). The relative swelling was then calculated as the average increase in particle size after wetting, based on the weight fraction of each excipient $\left(c_{i}\right)$.

$$
\Delta s=\sum_{i}^{N}\left(\frac{\Delta r_{\max }}{D_{50} / 2}\right) c_{i}
$$

\subsection{Tablet Manufacture}

\subsubsection{Investigating Formulation Effects}

The formulations manufactured are listed in Table 1. For each formulation, $300 \mathrm{~g}$ of blend was prepared by mixing both fillers $(47 \% \mathrm{w} / \mathrm{w}$ each) and the disintegrant $(5 \% \mathrm{w} / \mathrm{w})$ for $20 \mathrm{~min}$ in a blender (Pharmatech AB-015, Pharmatech, Warwickshire, UK) with a blend speed of $20 \mathrm{rpm}$ and an agitator speed of $200 \mathrm{rpm}$. Magnesium stearate $(1 \% \mathrm{w} / \mathrm{w})$ was then added and the mixture was blended for a further $5 \mathrm{~min}$.

Tablets were compacted with a $9 \mathrm{~mm}$ flat round die using a single punch automated tablet press (FlexiTab, Bosch Packaging Technology Ltd, Merseyside, UK). For each formulation, the filling depth and compression force were adjusted to obtain tablets with a target weight of approximately $350 \mathrm{mg}$ and target tensile strength of $>2.5 \mathrm{MPa}$. For MCC/mannitol, MCC/lactose and MCC/DCPA, tablets were compressed at $10 \mathrm{kN}$. For DCPA/lactose formulations, $16 \mathrm{kN}$ was used.

\subsubsection{Investigating Porosity and Disintegrant Con- centration Effects}

Additional tablets were manufactured for each filler combination with CCS (see Table 2). These tablets were manufactured with either higher porosity or higher disintegrant concentration.

To investigate the effect of porosity, the compression force was set to $8 \mathrm{kN}$ for $\mathrm{MCC} /$ mannitol, $\mathrm{MCC} /$ lactose and MCC/DCPA formulation. The DCPA/lactose formulation was compressed $12 \mathrm{kN}$. This decrease in compression force resulted in tablets with higher porosity than the original batches (see Section 2.3.1).

To investigate the effect of disintegrant concentration, additional tablets of these formulations were also prepared with $8 \% \mathrm{w} / \mathrm{w}$ of $\mathrm{CCS}$ at $10 \mathrm{kN}$ (MCC/mannitol, MCC/lactose and MCC/DCPA) and $16 \mathrm{kN}$ (DCPA/lactose). For tablets containing $8 \% \mathrm{w} / \mathrm{w} \mathrm{CCS}$, the concentration of magnesium
Table 1: Direct compression tablet formulations

\begin{tabular}{ccc}
\hline \hline Filler 1 & Filler 2 & Disintegrant \\
\hline MCC & Mannitol & CCS \\
MCC & Mannitol & XPVP \\
MCC & Mannitol & L-HPC \\
MCC & Mannitol & SSG \\
\hline MCC & Lactose & CCS \\
MCC & Lactose & XPVP \\
MCC & Lactose & L-HPC \\
MCC & Lactose & SSG \\
\hline MCC & DCPA & CCS \\
MCC & DCPA & XPVP \\
MCC & DCPA & L-HPC \\
MCC & DCPA & SSG \\
\hline DCPA & Lactose & CCS \\
DCPA & Lactose & XPVP \\
DCPA & Lactose & L-HPC \\
DCPA & Lactose & SSG \\
\hline
\end{tabular}

stearate was kept at $1 \% \mathrm{w} / \mathrm{w}$ and the quantity of each filler was adjusted to $45.5 \% \mathrm{w} / \mathrm{w}$ for each formulation.

\subsection{Characterisation of Tablets}

\subsubsection{Weight, Dimensions and Tensile Strength}

The weight of each tablet was measured to the nearest $0.1 \mathrm{mg}$ using an analytical balance. The diameter and thickness of each tablet were measured using a set of digital callipers and reported to the nearest $0.01 \mathrm{~mm}$.

The hardness of each tablet was measured using a hardness tester (Copley TBF 1000, Copley Scientific Ltd, Nottingham, UK). The tensile strength, $\sigma_{t}$, of tablets was calculated using the tablet hardness, $F$, diameter, $d$, and thickness, $t$ (Fell and Newton, 1970):

$$
\sigma_{t}=\frac{2 \cdot F}{\pi \cdot d \cdot t} .
$$

The weight, dimensions and tensile strength are reported as the mean of 10 tablets for each formulation.

\subsubsection{Porosity}

The porosity, $\varepsilon$, of tablets was calculated using the measured weight, $m$, dimensions and the true density, $\varrho_{t, \text { mix }}$, of the formulation. $\varrho_{t \text {,mix }}$ was calculated as the weighted harmonic mean considering the true density, $\varrho_{t, i}$, and the weight fraction, $c_{i}$, of each excipient (Sun et al., 2018): 
Table 2: Formulations used to manufacture additional tablets with higher porosity and higher disintegrant concentration

\begin{tabular}{lllll}
\hline \hline Filler 1 & Filler 2 & Disintegrant & $\begin{array}{l}\text { Compression } \\
\text { Forces }(\mathrm{kN})\end{array}$ & $\begin{array}{l}\text { Disintegrant Concen- } \\
\text { trations }(\% \mathrm{w} / \mathrm{w})\end{array}$ \\
\hline MCC & Mannitol & CCS & 8,10 & 5,8 \\
MCC & Lactose & CCS & 8,10 & 5,8 \\
MCC & DCPA & CCS & 8,10 & 5,8 \\
DCPA & Lactose & CCS & 12,16 & 5,8 \\
\hline
\end{tabular}

$$
\varrho_{t, \operatorname{mix}}=\left(\sum_{i}^{N} \frac{c_{i}}{\varrho_{t, i}}\right)^{-1}
$$

with $N=4$ as the number of different excipients in the formulation. The porosity can then be calculated using

$$
\varepsilon=1-\frac{\frac{m}{\pi \cdot(d / 2)^{2} \cdot t}}{\varrho_{t, \text { mix }}}
$$

The porosity was reported as the mean of 10 tablets for each formulation.

\subsubsection{Disintegration time}

The disintegration time of tablets was measured using a Copley DTG 2000 Disintegration Tester (Copley Scientific Ltd, Nottingham, UK). Tablets were disintegrated in $800 \mathrm{~mL}$ of distilled water at $37^{\circ} \mathrm{C}$. Disintegration time was measured in seconds for 6 tablets per formulation. The mean and standard deviation of these 6 tablets is reported.

\subsubsection{Dynamic Contact Angle}

Dynamic contact angle measurements were taken using a drop shape analyser (Krüss DSA30, Krüss GmbH, Hamburg, Germany). Video recordings were taken at a rate of 30 frames per second as a single droplet of MilliQ ${ }^{\circledR}$ Ultra-pure water was dispensed on to the surface of the tablet. The video files were analysed using MATLAB (R2019a, MathWorks, Massachusetts, USA) to determine the contact angle between the droplet and the tablet surface at each frame in the recording (see Fig S2 in the Supporting Information).

Two tablets were tested for each formulation. Using the data collected within the first second of contact with the liquid droplet for each pair of replicates, a two-phase exponential decay model (Eq. 5) was fitted to the data using GraphPad Prism 8 (version 8.3.1, GraphPad Software LLC, San Diego).

$$
\begin{aligned}
\theta_{c}(t) & =\theta_{c, p}+s_{f} e^{-k_{f} \cdot t}+s_{s} e^{-k_{s} \cdot t} \\
s_{f} & =\theta_{c, 0} \cdot x_{f s} \\
s_{s} & =\theta_{c, 0}\left(1-x_{f s}\right)
\end{aligned}
$$

$\theta_{c, 0}$ and $\theta_{c, p}$ are the contact angles at initial and infinite time, respectively. $k_{f}$ and $k_{s}$ are the rate constants for the fast and slow phases, respectively. The fraction of time dominated by the fast phase of the reaction is described as $x_{f s}$.

\subsubsection{Statistical Analysis}

To compare the effects of porosity and increased CCS concentration, individual t-tests were performed using GraphPad Prism 8 (version 8.3.1, GraphPad Software LLC, San Diego). Differences were considered statistically significant for $p<0.05$.

\section{Results}

\subsection{Characterisation of Raw Materials}

Moisture sorption isotherms of the fillers (Fig 1A) show that lactose, mannitol and DCPA are nonhygroscopic and do not absorb moisture, even at high relative humidity conditions. MCC is hygroscopic and absorbs a substantial amount of moisture.

For the disintegrants (Fig 1B), the moisture sorption isotherms show high hygroscopicity. For L$\mathrm{HPC}$, the moisture sorption at 80 and $90 \% \mathrm{RH}$ is lower than for the other excipients. For CCS, XPVP and SSG, each disintegrant absorbs approximately $42 \%$ moisture at $90 \% \mathrm{RH}$.

The results of particle size, sphericity and true density measurements for each excipient are given in Table 3. For all excipients the sphericity of the particles was $>0.6$. The smallest particle sizes were observed for SSG and CCS, whilst DCPA had the largest particle size. 

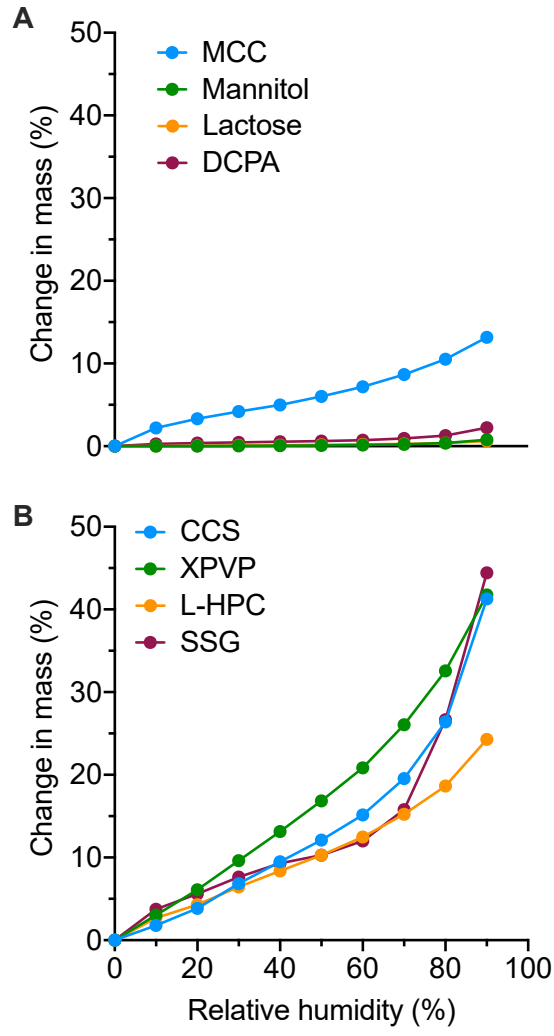

Figure 1: Moisture sorption isotherms for (A) fillers and (B) disintegrants.

Table 3: True density, particle size and sphericity of each excipient

\begin{tabular}{cccc}
\hline Excipient & $\varrho_{t}\left(\mathrm{~g} / \mathrm{cm}^{3}\right)$ & $D_{50}(\mu \mathrm{m})$ & $S_{50}$ \\
\hline MCC & 1.56 & 111.14 & 0.75 \\
Mannitol & 1.49 & 177.30 & 0.85 \\
Lactose & 1.55 & 130.76 & 0.85 \\
DCPA & 2.98 & 190.52 & 0.79 \\
CCS & 1.60 & 54.32 & 0.66 \\
XPVP & 1.25 & 105.07 & 0.80 \\
L-HPC & 1.48 & 79.03 & 0.63 \\
SSG & 1.55 & 53.59 & 0.87 \\
\hline
\end{tabular}

The measurement of single particle dissolution showed that mannitol particles dissolve in $12.5 \pm$ $7.2 \mathrm{~s}$, and lactose particles dissolve in $42.7 \pm 13.6$ s. Example images from the analysis are shown in Fig S1 in the Supporting Information.

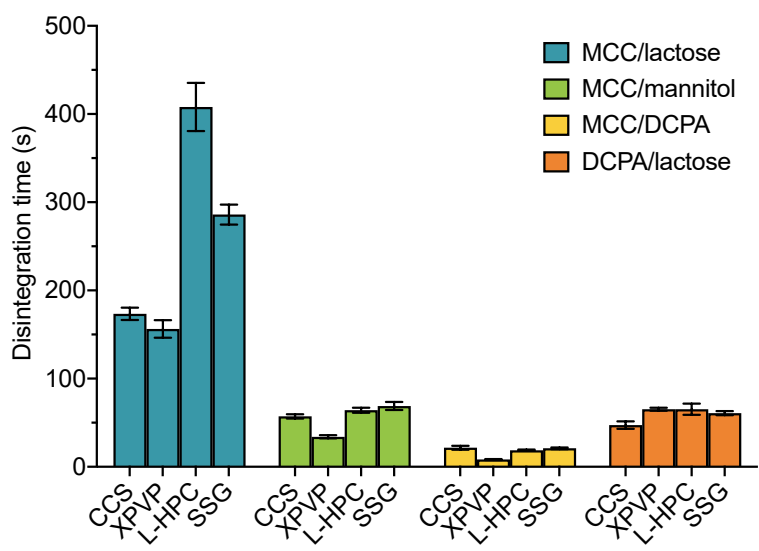

Figure 2: The disintegration time of each formulation $(n=6$, mean \pm standard deviation)

\subsection{Characterisation of Tablets}

\subsubsection{Tensile Strength and Porosity}

The tensile strength of all formulations was between 2.5 and 4.1 MPa (see Fig S3 in the Supporting Information). The porosity of tablets containing $\mathrm{MCC} / \mathrm{mannitol}$ and MCC/lactose was approximately $13 \%$, whilst the porosity of MCC/DCPA and DCPA/lactose tablets were around $23 \%$ and $19 \%$, respectively (See Fig S4 in the Supporting Information).

\subsubsection{Disintegration Time}

The disintegration times of each formulation is shown in Fig 2. Disintegration times were lowest for MCC/DCPA formulations. For MCC/mannitol, MCC/DCPA and DCPA/lactose, disintegration times were under 2 minutes for all disintegrants, whereas disintegration took significantly longer and large differences between the different disintegrants could be observed for MCC/lactose.

For MCC/lactose, $\mathrm{MCC} /$ mannitol and MCC/DCPA, the batches containing XPVP resulted in the fastest disintegration. For DCPA/lactose, the batch containing CCS disintegrated slightly faster.

\subsubsection{Dynamic Contact Angle}

The initial contact angle $\theta_{c, 0}$ was extracted from the dynamic contact angle measurements of the tablets (Fig 3). The difference between tablets with each disintegrant are small for MCC/DCPA, whereas they are more distinct for $\mathrm{MCC} /$ lactose and $\mathrm{MCC} /$ mannitol formulations. 


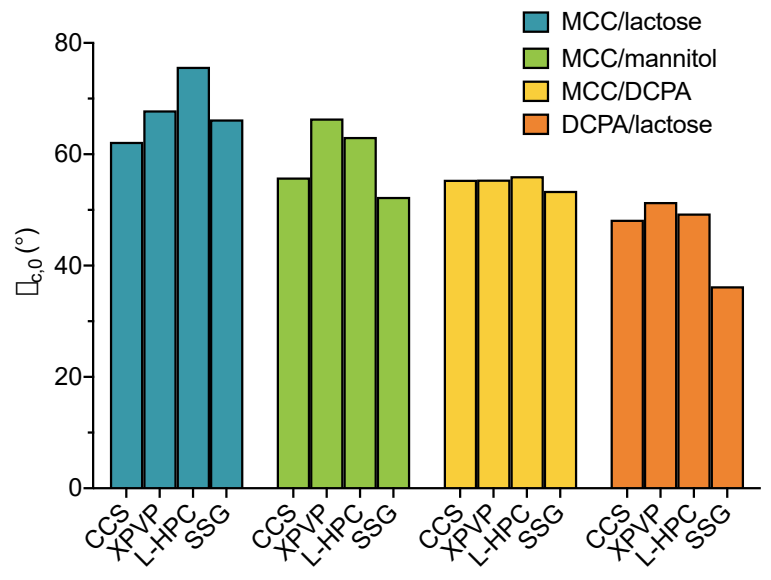

Figure 3: The initial contact angle of each formulation $(n=$ 2)

For DCPA/lactose, the contact angle is similar for CCS, XPVP and L-HPC, however $\theta_{c, 0}$ is much lower for tablets containing SSG. The full contact angle profiles are shown in Fig S5 in the Supporting Information.

\subsection{Investigating the Performance-Controlling Properties}

\subsubsection{Changing Tablet Porosity}

Additional tablets were manufactured for each filler-combination with CCS to investigate the effect of changing the tablet porosity. The porosity and disintegration time of the new tablets are shown against the original tablets in Fig 4. When the porosity was increased, the disintegration time of MCC/lactose decreased significantly. This suggests that the disintegration time was limited by liquid penetration. On the other hand, the disintegration of tablets containing $\mathrm{MCC} /$ mannitol was much slower when the porosity was increased. For MCC/DCPA and DCPA/lactose, increasing the porosity did not significantly change the disintegration times.

\subsubsection{Changing Disintegrant Concentration}

The disintegration times of batches with $5 \%$ and $8 \% \mathrm{w} / \mathrm{w}$ CCS are shown in Fig 5. When the disintegrant concentration was increased, the disintegration times of all tablets changed. For $\mathrm{MCC} /$ lactose, an increase in CCS concentration resulted in a faster tablet disintegration. There was a slight increase in disintegration time observed for $\mathrm{MCC} /$ mannitol and DCPA-based formulations.

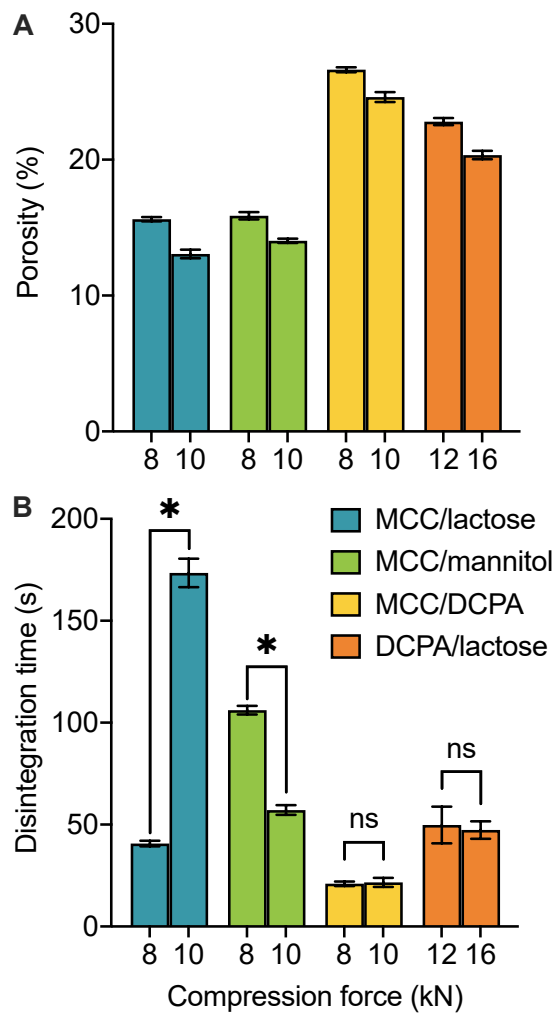

Figure 4: The (A) porosity and (B) disintegration time of tablets compressed at 8 and $10 \mathrm{kN}$ (MCC/lactose, $\mathrm{MCC} / \mathrm{mannitol}$ and $\mathrm{MCC} / \mathrm{DCPA}$ ) or 12 and $16 \mathrm{kN}$ (DCPA/lactose). $n=$ (A) 10 or (B) 6 ; mean \pm standard deviation; ${ }^{*} p<0.05$; ns: not significant.

The initial contact angle of tablets containing $5 \%$ and $8 \% \mathrm{w} / \mathrm{w}$ CCS are shown in Fig 6. For tablets containing $\mathrm{MCC} /$ lactose, $\mathrm{MCC} /$ mannitol and MCC/DCPA, there was a decrease in initial contact angle for tablets containing $8 \% \mathrm{w} / \mathrm{w}$ CCS. For tablets containing DCPA/lactose, the initial contact angle increased with higher disintegrant concentration.

\section{Discussion}

\subsection{The Influence of Formulation on Disintegra- tion}

The performance of IR tablets is controlled by different disintegration mechanisms. These mechanisms can be impacted by both raw material properties and the manufacturing conditions. The key differences between the raw material and tablet 


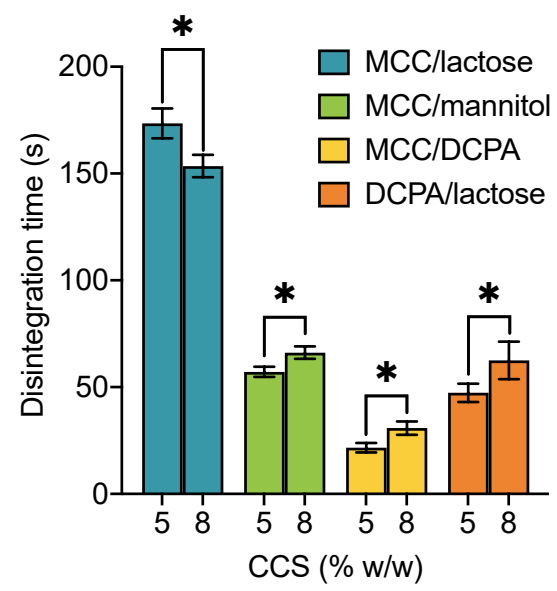

Figure 5: Disintegration times of batches prepared with $5 \%$ and $8 \% \mathrm{w} / \mathrm{w}$ CCS $\left(n=6 ;\right.$ mean \pm standard deviation; ${ }^{*} p<$ 0.05 )

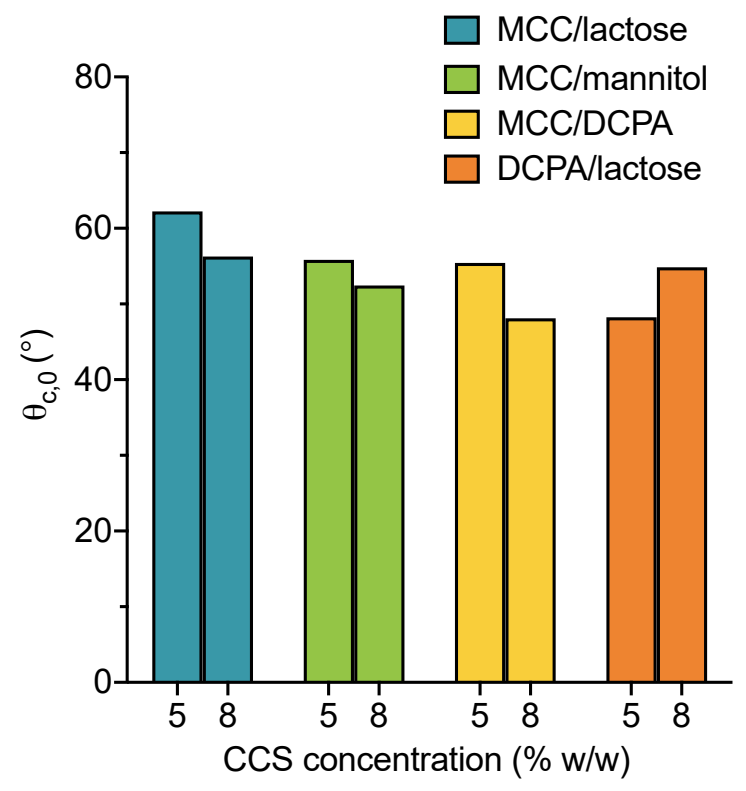

Figure 6: The initial contact angle, $\theta_{c, 0}$, of tablets containing 5 and $8 \% \mathrm{w} / \mathrm{w} \operatorname{CCS}(n=2$ and $n=4$, for 5 and $8 \% \mathrm{w} / \mathrm{w}$ CCS, respectively.

properties of tablets containing different filler combinations with $5 \% \mathrm{w} / \mathrm{w}$ CCS are shown in Fig 7 and the workflow used to classify these formulations is shown in Fig 8.

For all tablets, liquid penetration is the necessary pre-requisite for disintegration and dissolution. As such, ensuring rapid liquid penetration will al-

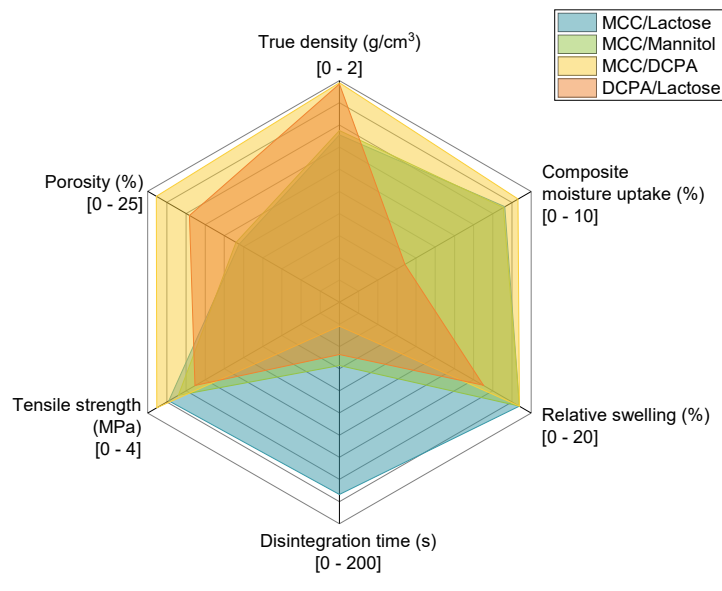

Figure 7: Summary of raw material and tablet properties for each filler combination with $5 \% \mathrm{w} / \mathrm{w}$ CCS. Raw material properties $\left(\varrho_{t, \text { mix }}\right.$, relative swelling and moisture uptake) expressed as the weighted arithmetic mean of each excipient.

ways be a key consideration during the development of directly compressed tablets. In this study, each batch has been further classified based on the disintegration-controlling mechanisms. This allows the formulator to identify the properties which can be adjusted slightly in order to optimise the disintegration performance. For example, the DCPAbased tablets tested in Section 3.3.1 show that increasing the porosity of these tablets (as a result, increasing the rate of liquid penetration) did not result in faster disintegration (Fig 4). However, changing the disintegrant concentration had a significant effect on disintegration time (Fig 5). This indicates that for the swelling-controlled DCPAbatches, disintegration can be optimised by careful selection of the disintegrant and concentration.

The first stage in the workflow is to assess the solubility of the major tablet components. Several studies in the literature have demonstrated that disintegration is faster for tablets composed of insoluble excipients compared to those with soluble excipients (Rubinstein and Birch, 1977; Johnson et al., 1991). The disintegration times of these batches (shown in Fig 2) support these findings, as tablets containing MCC and DCPA disintegrate faster than those containing mannitol or lactose. If the tablet matrix is mostly composed of a soluble material, then liquid penetration will lead to the dissolution of filler particles. For tablets composed of an insoluble filler, only a small amount of water will be used to wet the filler particles, thus leaving more water 


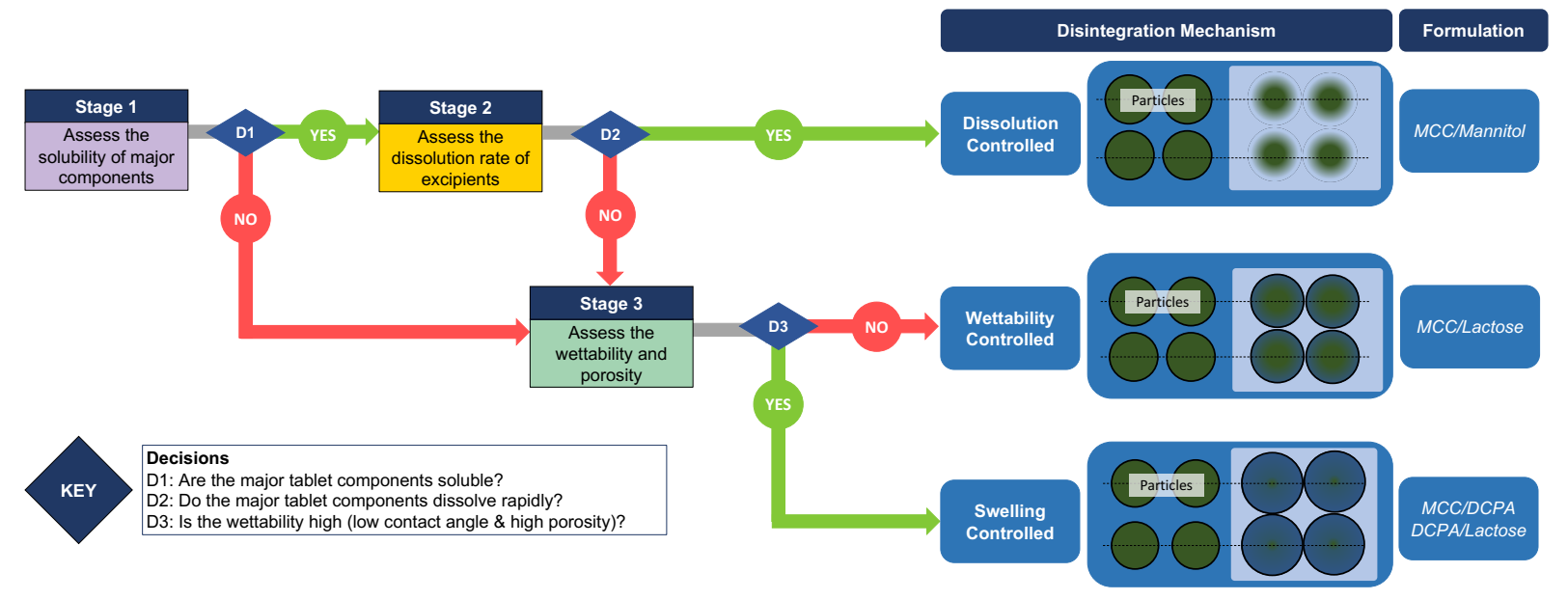

Figure 8: Workflow for the classification of IR tablets based on the disintegration mechanism.

available for the disintegrant (Ekmekciyan et al., 2018). The solubility of most pharmaceutical materials is well-defined in the literature.

In addition to the solubility of the tablet components, it is also important to consider the intrinsic dissolution rate of the excipients in order to distinguish between components which will dissolve rapidly when in contact with liquid and those which will dissolve more slowly. For example, lactose and mannitol have very similar absolute solubility values $(1 \mathrm{~g}$ in $5.24 \mathrm{~mL}$ and $1 \mathrm{~g}$ in $5.5 \mathrm{~mL}$, respectively, for water at $20^{\circ} \mathrm{C}$ (Rowe et al., 2009)), however, the rates at which these excipients dissolve are different. This was previously shown through determining the intrinsic dissolution rates by Wewers et al. (2020), and further supported by the single particle dissolution analysis performed for lactose and mannitol (Section 3.1). The difference in filler dissolution rate is evident when comparing the disintegration time of tablets containing $\mathrm{MCC} / \mathrm{mannitol}$ and MCC/lactose (Fig 2). When mannitol is used as a filler, these particles will dissolve rapidly from the matrix, resulting in an increase in the apparent pore space and improved liquid penetration. On the other hand, lactose particles will take longer to dissolve, meaning that the porosity remains low during the initial stages of disintegration. If both the solubility and intrinsic dissolution rate are high for the major tablet components, then disintegration is driven by the dissolution of particles from the matrix which consequently leads to improved liquid penetration. The disintegration mechanism of $\mathrm{MCC} /$ mannitol is thus dissolution controlled.

The third stage in the workflow is to assess the wettability and porosity of the tablets. The porosity of the tablets strongly influences the performance as it relates directly to the rate of liquid penetration. The total porosity of the tablet includes both intra- and inter-particle porosity. DCPA has a high intra-particle porosity, meaning that tablets composed of MCC/DCPA and DCPA/lactose have much higher total porosity than those containing MCC/lactose and MCC/mannitol (see Fig S4 in the Supporting Information). This high porosity promotes liquid penetration through the pores in the tablets, which then allows other processes such as swelling or dissolution to begin. Due to the rapid liquid penetration and wetting, disintegration is limited by the rate of swelling of the disintegrant particles. On the other hand, tablets containing MCC/lactose have much lower porosity (Fig S4 in the Supporting Information) than those containing DCPA. Unlike the tablets containing mannitol (in which mannitol particles dissolve rapidly upon contact with liquid), the single particle dissolution analysis has shown that the wetting and dissolution of lactose particles is much slower. As a result, the porosity of this batch will remain low during the beginning of disintegration, leading to slow liquid penetration and delayed swelling compared to the DCPA-based batches. We have therefore classified the MCC/lactose batch as wettability-controlled.

The swelling ability of a formulation is determined by the swelling capacity of the raw materials 
(MCC, CCS, L-HPC, SSG) as well as shape recovery effects (XPVP). For the MCC-based formulations, both the disintegrant and MCC contribute to the swelling (both omni- and uni-directional, for swelling and shape recovery excipients respectively) within a tablet. The swelling of the DCPA/lactose formulation is only driven by the disintegrant used. $\mathrm{MCC} / \mathrm{DCPA}$ and DCPA/lactose are both swellingcontrolled, whereas the MCC/DCPA formulations disintegrate faster due to its higher swelling ability as exemplified by the relative swelling, $\Delta s$, in Fig 7.

Each filler combination was manufactured with four different disintegrants: CCS, XPVP, L-HPC and SSG. The difference in disintegration times between the disintegrants were relatively small for tablets composed of MCC/mannitol, MCC/DCPA and DCPA/lactose. On the other hand, disintegrant choice played a crucial role in determining the rate of disintegration of tablets containing MCC/lactose. For these batches, XPVP resulted in the fastest disintegration, followed by CCS, SSG and L-HPC. These findings are in agreement with previous studies which have ranked the effectiveness of different disintegrants. Specifically, Quodbach et al. (2014a) studied the development of fractal dimensions for dibasic calcium phosphate (DCP) based tablets containing different disintegrants. The results of this study showed that XPVP was the most effective disintegrant, followed by CCS and SSG (Quodbach et al., 2014a).

The workflow shown in Fig 8 describes a classification system to determine the performancecontrolling disintegration mechanism of a tablet. This workflow was developed based on the formulations used in this study. In order to fully explore the design space and establish criteria for the decision points in this workflow, further research is required to assess the influence of changing the composition or excipients, as well as the effects of API.

\subsection{The Influence of Porosity on Disintegration}

Additional batches were manufactured for each filler combination with CCS in order to investigate the effect of porosity on the disintegration mechanisms. Tablets containing MCC/DCPA and DCPA/lactose showed no significant difference in disintegration time when manufactured at low and high porosity (as shown in Fig 4). This supports the conclusion that swelling, rather than liquid penetration, is the limiting step in the disintegration of these tablets.
The disintegration of MCC/mannitol tablets was slower for the batch of tablets with high porosity. For tablet disintegration to occur, disintegrants must exert a sufficient swelling force against the surrounding matrix. Liquid penetration of these tablets results in the rapid dissolution of mannitol. As mannitol dissolves, the apparent pore space in the tablet increases. If the empty pore space between the disintegrant and MCC particles is too large, then particles will swell into this space instead of exerting a force against the surrounding particles. This could cause an increase in disintegration time, as the swelling mechanism of the disintegrant and MCC is less efficient.

The disintegration time of MCC/lactose tablets was significantly faster for tablets manufactured at the lower compression force (i.e. higher porosity tablets). This confirms the conclusion that these tablets are wettability-controlled, as increased porosity accelerated the liquid uptake and, consequently, reduced the disintegration time.

\subsection{The Influence of Disintegrant Concentration on Disintegration}

The effect of disintegrant concentration was also investigated for each filler combination. Tablets were manufactured with $8 \% \mathrm{w} / \mathrm{w}$ CCS to allow comparison with the original batches containing $5 \%$ CCS.

When the disintegrant concentration was increased, the disintegration time of MCC/DCPA and DCPA/lactose tablets increased (Fig 5). Similar results were also found by Berardi et al. (2018), with DCPA-based tablets showing slower disintegration with higher concentrations of CCS or SSG. The authors attributed these results to the formation of a hydrated gel matrix, which held the tablet together despite increased liquid uptake and swelling. It is possible that at $8 \%$ CCS a gel is formed that results in a barrier preventing further liquid penetration.

Tablets containing MCC/mannitol also showed a slight increase in disintegration time when the concentration of CCS was increased. Despite the higher wettability (as shown by a lower initial contact angle in Fig 6), these tablets also showed lower porosity and higher tensile strength compared to those containing $5 \% \mathrm{w} / \mathrm{w}$ CCS. If disintegration of these tablets is controlled by dissolution of mannitol particles, then the lower porosity could impede liquid penetration and result in delayed dissolution 
of the mannitol. On the other hand, tablets composed of MCC/lactose show faster disintegration when $8 \% \mathrm{w} / \mathrm{w}$ CCS is used instead of $5 \% \mathrm{w} / \mathrm{w}$. As shown in Fig 6, a decrease in initial contact angle for this batch suggests increased wettability. Unlike tablets containing $\mathrm{MCC} / \mathrm{mannitol}$, there is no effect on porosity (Fig S6 in the Supporting Information). As a result, the increase in wettability accelerates liquid uptake and hence decreases the disintegration time.

\section{Conclusions}

The performance of tablets containing different formulations, porosities and compositions were investigated. This work provides a comprehensive explanation of the mechanisms which control disintegration time for tablets with different raw material and compact properties. The processes which may control disintegration are wettability (i.e. liquid penetration), swelling or dissolution. The mechanism of disintegration for a formulation may be determined by considering the properties of the raw materials. For fillers which contain a high proportion of rapidly-dissolving material, disintegration will likely be dissolution controlled. If the excipient wettability is low and the tablets have low porosity, disintegration will be controlled by the liquid penetration. For tablets which are insoluble or partially insoluble, swelling may be the controlling factor. These mechanisms were further supported by investigating the effect of increasing the tablet porosity. A $2 \%$ change in porosity for the wettability-controlled tablets (MCC/lactose) caused a $77 \%$ reduction in the disintegration time, whereas for swelling controlled tablets (MCC/DCPA and DCPA/lactose) a change in porosity of $2 \%$ (MCC/DCPA) and $7 \%$ (DCPA/lactose) did not significantly change the disintegration process $(3 \%$ and $5 \%$ change in disintegration time, respectively). The use of the simple workflow presented in this study could allow formulators to identify the key formulation or manufacturing parameters which could be adjusted to optimise disintegration.

In this study, placebo tablets were used to investigate the effects of different excipients, but the effect of API must also be assessed when considering the performance-controlling mechanism of a formulation. Moreover, the composition of the tablets is likely to have a strong influence on the disintegration controlling mechanism, and so changes to the ratio of fillers or disintegrant must be taken into account. In addition to the formulation, the choice of dissolution medium (e.g. biorelevant medium) and its temperature can affect the disintegration and dissolution. Generating a full picture of the relationship between given raw materials, manufacturing conditions and controlling disintegration mechanism will require the consideration of biorelevant dissolution media.

\section{Acknowledgements}

We would like to acknowledge the U.K. Engineering and Physical Sciences Research Council (EP$\mathrm{SRC}$ ) and AstraZeneca for funding.

\section{References}

Al-Sharabi, M., Markl, D., Mudley, T., Bawuah, P., Karttunen, A.P., Ridgway, C., Gane, P., Ketolainen, J., Peiponen, K.E., Rades, T., Zeitler, J.A., 2020. Simultaneous investigation of the liquid transport and swelling performance during tablet disintegration. Int. J. Pharm. 584, 119380. doi:10.1016/j.ijpharm.2020.119380.

Anwar, S., Fell, J.T., Dickinson, P.A., 2005. An investigation of the disintegration of tablets in biorelevant media. Int. J. Pharm. 290, 121-127. doi:10.1016/j.ipharm.2004.11.023.

Basaleh, S., Bisharat, L., Cespi, M., Berardi, A., 2020. Temperature: An overlooked factor in tablet disintegration. Eur. J. Pharm. Sci. 151, 1-9. doi:10.1016/j.ejps.2020.105388.

Berardi, A., Bisharat, L., Blaibleh, A., Pavoni, L., Cespi, M., 2018. A simple and inexpensive image analysis technique to study the effect of disintegrants concentration and diluents type on disintegration. J. Pharm. Sci. 107, 2643-2652. doi:10.1016/j.xphs.2018.06.008.

Bisharat, L., AlKhatib, H.S., Muhaissen, S., Quodbach, J., Blaibleh, A., Cespi, M., Berardi, A., 2019. The influence of ethanol on superdisintegrants and on tablets disintegration. Eur. J. Pharm. Sci. 129, 140-147. doi:10.1016/j.ejps.2019.01.004.

Caramella, C., Ferrari, F., Bonferoni, M.C., Ronchi, M., 1990. Disintegrants in solid dosage forms. Drug Dev. Ind. Pharm. 16, 2561-2577. doi:10.3109/03639049009058547.

Desai, P.M., Liew, C.V., Heng, P.W., 2012. Understanding disintegrant action by visualization. J. Pharm. Sci. 101, 2155-64. doi:10.1002/jps.23119.

Desai, P.M., Liew, C.V., Heng, P.W.S., 2016. Review of disintegrants and the disintegration phenomena. J. Pharm. Sci. 105, 2545-2555. doi:10.1016/j.xphs.2015.12.019.

Dvořák, J., Tomas, J., Lizoňová, D., Schöngut, M., Dammer, O., Pekárek, T., Beránek, J., Stepanek, F., 2020. Investigation of tablet disintegration pathways by the combined use of magnetic resonance imaging, texture analysis and static light scattering. Int. J. Pharm. 587, 119719. doi:10.1016/j.ijpharm.2020.119719.

Ekmekciyan, N., Tuglu, T., El-Saleh, F., Muehlenfeld, C., Stoyanov, E., Quodbach, J., 2018. Competing for water: A new approach to understand disintegrant performance. Int. J. Pharm. 548, 491-499. doi:10.1016/j.ijpharm.2018.07.025. 
Fell, J.T., Newton, J.M., 1970. Determination of tablet strength by the diametral-compression test. J. Pharm. Sci. 59, 688-691. doi:10.1002/jps.2600590523.

Gordon, M.S., Chowhan, Z.T., 1987. Effect of tablet solubility and hygroscopicity on disintegrant efficiency in direct compression tablets in terms of dissolution. J. Pharm. Sci. 76, 907-909. doi:10.1002/jps.2600761213.

Gordon, M.S., Rudraraju, V.S., Dani, K., Chowhan, Z.T., 1993. Effect of the mode of super disintegrant incorporation on dissolution in wet granulated tablets. J. Pharm. Sci. 82, 220-226. doi:10.1002/jps.2600820222.

Johnson, J., Wang, L., Gordon, M.S., Chowhan, Z.T., 1991. Effect of formulation solubility and hygroscopicity on disintegrant efficiency in tablets prepared by wet granulation, in terms of dissolution. J. Pharm. Sci. 80, 469-471. doi:10.1002/jps.2600800514.

López-Solís, J., Villafuerte-Robles, L., 2001. Effect of disintegrants with different hygroscopicity on dissolution of norfloxacin/pharmatose dcl 11 tablets. Int. J. Pharm. 216, 127-135. doi:10.1016/s0378-5173(01)00584-1.

Marais, A.F., Song, M., De Villiers, M.M., 2003. Effect of compression force, humidity and disintegrant concentration on the disintegration and dissolution of directly compressed furosemide tablets using croscarmellose sodium as disintegrant. Trop. J. Pharm. Res. 2. doi:10.4314/tjpr.v2i1.14577.

Markl, D., Yassin, S., Wilson, D.I., Goodwin, D.J., Anderson, A., Zeitler, J.A., 2017. Mathematical modelling of liquid transport in swelling pharmaceutical immediate release tablets. Int. J. Pharm. doi:10.1016/j.ijpharm.2017.04.015.

Markl, D., Zeitler, J.A., 2017. A Review of Disintegration Mechanisms and Measurement Techniques. Pharm. Res. 34, 890-917. doi:10.1016/j.ijpharm.2004.11.023.

Nogami, H., Hasegawa, J., Miyamoto, M., 1967. Studies on powdered preparations. xx. disintegration of the aspirin tablets containing starches as disintegrating agent. Chem. Pharm. Bull. 15, 279-289. doi:10.1248/cpb.15.279.

O'Mahoney, N., Keating, J.J., McSweeney, S., Hill, S., Lawrence, S., Fitzpatrick, D., 2020. The sound of tablets during coating erosion, disintegration, deaggregation and dissolution. Int. J. Pharm. 580, 119216. doi:10.1016/j.ijpharm.2020.119216.

Patel, N.R., Hopponen, R.E., 1966. Mechanism of action of starch as a disintegrating agent in aspirin tablets. J. Pharm. Sci. 55, 1065-1068. doi:10.1002/jps.2600551015.

Quodbach, J., Kleinebudde, P., 2016. A critical review on tablet disintegration. Pharm. Dev. Technol. 21, 763-774. doi:10.3109/10837450.2015.1045618.

Quodbach, J., Moussavi, A., Tammer, R., Frahm, J. Kleinebudde, P., 2014a. Assessment of disintegrant efficacy with fractal dimensions from real-time mri. Int. J. Pharm. 475, 605-612. doi:10.1016/j.ijpharm.2014.09.021.

Quodbach, J., Moussavi, A., Tammer, R., Frahm, J., Kleinebudde, P., 2014b. Tablet disintegration studied by high-resolution real-time magnetic resonance imaging. J. Pharm. Sci. 103, 249-255. doi:10.1002/jps.23789.

Rowe, R.C., Sheskey, P.J., Quinn, M.E., 2009. Handbook of Pharmaceutical Excipients. 6th ed., Pharmaceutical Press, London.

Rubinstein, M.H., Birch, M., 1977. The effect of excipient solubility on the in-vitro and in-vivo properties of bendrofluazide tablets $5 \mathrm{mg}$. Drug Dev. Ind. Pharm. 3, 439450. doi:10.3109/03639047709055623.

Soundaranathan, M., Vivattanaseth, P., Walsh, E., Pitt, K.,
Johnston, B., Markl, D., 2020. Quantification of swelling characteristics of pharmaceutical particles. Int J Pharm , 119903doi:10.1016/j.ijpharm.2020.119903.

Sun, W.J., Rantanen, J., Sun, C.C., 2018. Ribbon density and milling parameters that determine fines fraction in a dry granulation. Powder Technol. 338, 162-167. doi:10.1016/j.powtec.2018.07.009

Tomas, J., Schngut, M., Dammer, O., Bernek, J., Zadrail, A., tpnek, F., 2018. Probing the early stages of tablet disintegration by stress relaxation measurement. Eur. J. Pharm. Sci. 124, 145-152. doi:10.1016/j.ejps.2018.08.029.

Wewers, M., Czyz, S., Finke, J.H., John, E., Van Eerdenbrugh, B., Juhnke, M., Bunjes, H., Kwade, A., 2020. Influence of formulation parameters on redispersibility of naproxen nanoparticles from granules produced in a fluidized bed process. Pharm. 12. doi:10.3390/pharmaceutics12040363.

Yassin, S., Goodwin, D.J., Anderson, A., Sibik, J., Wilson, D.I., Gladden, L.F., Zeitler, J.A., 2015. The disintegration process in microcrystalline cellulose based tablets, part 1: Influence of temperature, porosity and superdisintegrants. J. Pharm. Sci. 104, 3440-3450. doi:10.1002/jps.24544.

Zhao, N., Augsburger, L.L., 2005. The influence of swelling capacity of superdisintegrants in different ph media on the dissolution of hydrochlorothiazide from directly compressed tablets. AAPS PharmSciTech 6, E120-E126. doi:10.1208/pt060119. 